\title{
Numerical Study of the Short Crack Initiation in Polycrystalline Aggregates
}

\author{
Yoann Guilhem \\ EDF R\&D/MMC \\ Avenue des Renardières, \\ Écuelles, F-77818 Moret-sur-Loing \\ France \\ Georges Cailletaud \\ Centre des Matériaux, CNRS UMR 7633 \\ MINES ParisTech \\ BP 87, F-91003 Evry Cedex \\ France
}

\author{
Jean-Michel Stéphan \& François Curtit \\ EDF R\&D/MMC \\ Avenue des Renardières, \\ Écuelles, F-77818 Moret-sur-Loing \\ France
}

Stéphanie Basseville

Laboratoire des Systèmes d'Ingénierie de Versailles

Université de Versailles

45 avenue des États-Unis, F-78035 Versailles

France

March 3, 2018

\begin{abstract}
Fatigue life of polycrystalline materials is mainly governed by the early stages of surface short cracks. Initiation and micro-propagation phases, which represent from $50 \%$ to $80 \%$ of total component life, are experimentally proven to depend on material microstructure. The aim of this numerical study is to highlight the influence of local parameters (e.g. crystallographic orientation) on the short crack initiation. Finite element computations are performed with a crystal plasticity model. The investigations are carried out on 316L Stainless Steel with different fatigue loadings and various surface roughnesses. A qualitative study of volumetric strain localization is carried out to distinguish uniaxial and biaxial results. The slip plane activity in surface grains is characterized. Many configurations are tested to provide a statistical point of view. Results revealed the strong vulnerability of surface grains with a high Schmid factor on systems that produce extrusions. It is also demonstrated that taking account of geometrical singularities can strongly change the plastic strain localization due to crystal orientation.
\end{abstract}

\section{NOMENCLATURE}

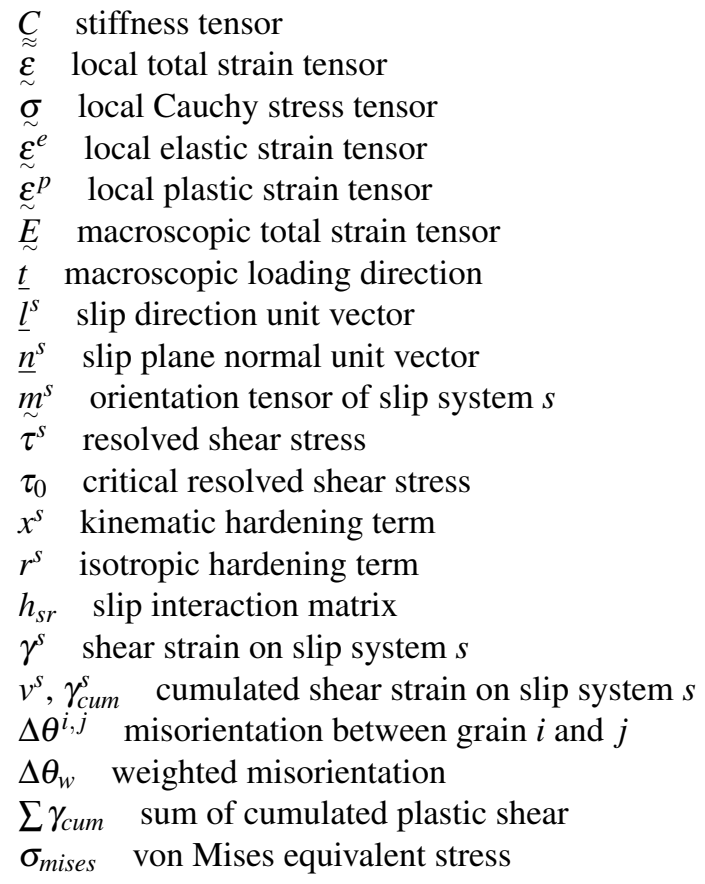


$\langle\bullet\rangle_{g} \bullet$ grain average

$\langle\bullet\rangle_{g}^{*} \quad \bullet$ grain second order momentum

$V_{g}$ grain volume

\section{INTRODUCTION}

The understanding of short fatigue cracks initiation and micro-propagation mechanisms is motivated by thermomechanical fatigue occurring in mixing zones of power plants cooling systems, for instance. In polycrystalline materials loaded under cyclic loading, Persistent Slip Bands (PSB) are formed by shear and extrusion steps appear at the specimen surface [1, 2]. These are often the sites of fatigue crack initiation when oxidation and decohesion occur at the base of the extrusion. Experimental observations using Electron Back Scattering Diffraction (EBSD) technique [3, 4] and Atomic Force Microscope (AFM) [5, 6] give accurate information about the influence of local microstructure features on micro-crack nucleation. However, these are only superficial analysis and a 3D volume analysis is needed. Recent improvements in tomography coupled with diffraction allow access to polycrystal grain volumetric shape and orientation [7, 8]. A wider set of local data is then available to allow a better comprehension of fatigue crack evolution in polycrystals. Unfortunately, this technique requires cutting edge facilities and is still restricted to few materials with specific grain sizes and chemical compositions.

Discrete Dislocation Dynamics [9] is used for studies on single crystals. Crystal plasticity laws are introduced into Finite Element codes to compute 3D polycrystalline aggregates [10, 11]. In a previous study, a simplistic 2D model has been used to investigate a wide range of local orientation configurations and to exhibit the cluster effect [12]. Explicit crack were introduced inside 2D mesh to analyze Crack Tip Opening Displacement depending on grain orientation [13, 14]. More complex 3D models are now used to give a statistical point of view over fatigue crack initiation in polycrystalline aggregates [15].

The aim of the study is to proceed to many polycrystalline aggregate FE computations with different loading conditions and various crystallographic orientation sets. We will also provide a statistical analysis on the influence of local microstructure features, such as crystallographic orientation and misorientation, on local plasticity which mainly governs fatigue crack initiation. The paper is organized as follows. First, the numerical model is presented with crystal plasticity equations, FE mesh, boundary conditions, specific parameters and variables. Then, the results are presented, with investigations on different mesh sizes, various loadings, multiple orientation realizations and surface roughness.

\section{DESCRIPTION OF THE MODEL}

\subsection{Constitutive equations}

Each grain is defined by a group of elements with a common crystallographic orientation. Displacements are assumed to be continuous at grain boundaries. Thus, it can be the place for stress discontinuities due to elastic and plastic anisotropy.

A phenomenological crystal plasticity model is implemented in the finite element code Zebulon [16]. At low strain amplitude, elastic anisotropy plays a preponderant role in local variables heterogeneities [17]. Anisotropic elasticity matrix is introduced for each grain to govern elastic strains by means of Hooke's law. Taking account of $316 \mathrm{~L}$ crystalline structure, in which austenitic phase prevails, cubic stiffness tensor $\underset{\approx}{C}$, defined by 3 coefficients $C_{1111}, C_{1122}$ and $C_{1212}$, is chosen. Strain rate is partitioned into elastic and viscoplastic part:

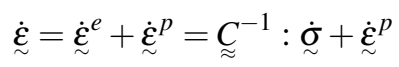

The resolved shear stress $\tau^{s}$ is computed on each slip system $s$ by means of the orientation tensor ${\underset{\sim}{m}}^{s}, \underline{n}^{s}$ being the normal to the slip plane, and $\underline{l}^{s}$ the slip direction.

$$
\begin{gathered}
\tau^{s}=\underset{\sim}{\sigma}: \underline{\sim}^{s} \\
\text { with }{\underset{\sim}{m}}^{s}=\frac{1}{2}\left(\underline{l}^{s} \otimes \underline{n}^{s}+\underline{n}^{s} \otimes \underline{l}^{s}\right)
\end{gathered}
$$

A Norton flow rule gives each viscoplastic slip rate $\dot{\gamma}^{s}$, which is combined with orientation tensor $m_{\sim}^{s}$. These 
terms are summed to get the viscoplastic strain rate tensor.

$$
\begin{gathered}
\dot{v}^{s}=\left\langle\frac{\left|\tau^{s}-x^{s}\right|-\tau_{0}-r^{s}}{K}\right\rangle^{n} \text { with }\langle\bullet\rangle=\left\{\begin{array}{l}
\bullet \text { if } \bullet \geq 0 \\
0 \text { if } \bullet<0
\end{array}\right. \\
\dot{\gamma}^{s}=\dot{v}^{s} \operatorname{sign}\left(\tau^{s}-x^{s}\right) \\
\dot{\sim}^{p}=\sum_{s} \dot{\gamma}_{\sim}^{s} m^{s}
\end{gathered}
$$

where $K$ and $n$ are the parameters which define viscosity, $\tau_{0}$ is the critical resolved shear stress, $x^{s}$ and $r^{s}$ are respectively the kinematic hardening and the isotropic hardening variables. The state variables $\alpha^{s}$, respectively $\rho^{s}$, control kinematic hardening term $x^{s}$, respectively isotropic hardening term $r^{s}$, through the following nonlinear relations.

$$
\begin{gathered}
x^{s}=c \alpha^{s} \\
r^{s}=b Q \sum_{r} h_{s r} \rho^{r} \\
\dot{\alpha}^{s}=\left(\operatorname{sign}\left(\tau^{s}-x^{s}\right)-d \alpha^{s}\right) \dot{v}^{s} \\
\dot{\rho}^{s}=\left(1-b \rho^{s}\right) \dot{v}^{s}
\end{gathered}
$$

Material parameters related to hardening are $c$ and $d$ for kinematic. $Q, b$ are isotropic hardening parameters and $h$ matrix defines interactions between different slip systems as defined by Franciosi [18].

Our investigation are carried out on $316 \mathrm{~L}$ Stainless Steel. Macroscopic behavior of the material is isotropic and it does not exhibit preferential orientation texture, that is why grain orientations are assigned with random Euler angles sets. The material is mainly composed of austenitic grains with Face Centred Cubic crystalline structure. Octahedral slip systems, i.e. $\{111\}\langle 110\rangle$ slip system family, are then involved in plastic glide mechanisms. The corresponding interaction matrix coefficients are fixed as a compromise between recent numerical identifications [19, 20]. Remaining single crystal material parameters are identified on cyclic uniaxial compressiontension tests by means of scale transition model called $\beta$-model [21]. The model is composed of 40 crystallographic orientations, providing an isotropic behavior [22]. It is assumed that $10^{\text {th }}$ simulated cycle is equivalent to experimental stabilized cycle. The values obtained are listed in table 1 and the comparison between experimental and numerical is presented in figure 1 which shows a good agreement.

\begin{tabular}{lc}
\hline \multicolumn{2}{c}{ Cubic elasticity } \\
\hline$C_{1111}$ & $197,000 \mathrm{MPa}$ \\
$C_{1122}$ & $125,000 \mathrm{MPa}$ \\
$C_{1212}$ & $122,000 \mathrm{MPa}$ \\
\hline \multicolumn{2}{c}{ Flow rule } \\
\hline$K$ & \multicolumn{1}{c}{$12 \mathrm{MPa} . \mathrm{s}^{-n}$} \\
$n$ & \multicolumn{1}{c}{11} \\
$\tau_{0}$ & \multicolumn{1}{c}{$40 \mathrm{MPa}$} \\
\hline Kinematic hardening \\
\hline$c$ & $40,000 \mathrm{MPa}$ \\
$d$ & \multicolumn{1}{c}{$1,500 \mathrm{MPa}$} \\
\hline \hline \multicolumn{2}{c}{ Isotropic hardening } \\
\hline$Q$ & $10 \mathrm{MPa}$ \\
$b$ & 3 \\
\hline Slip interaction matrix \\
\hline$h_{1}$ & 1 \\
$h_{2}$ & 1 \\
$h_{3}$ & 0.6 \\
$h_{4}$ & 12.3 \\
$h_{5}$ & 1.6 \\
$h_{6}$ & 1.8 \\
\hline
\end{tabular}

Table 1: Material parameters for 316L Stainless Steel.

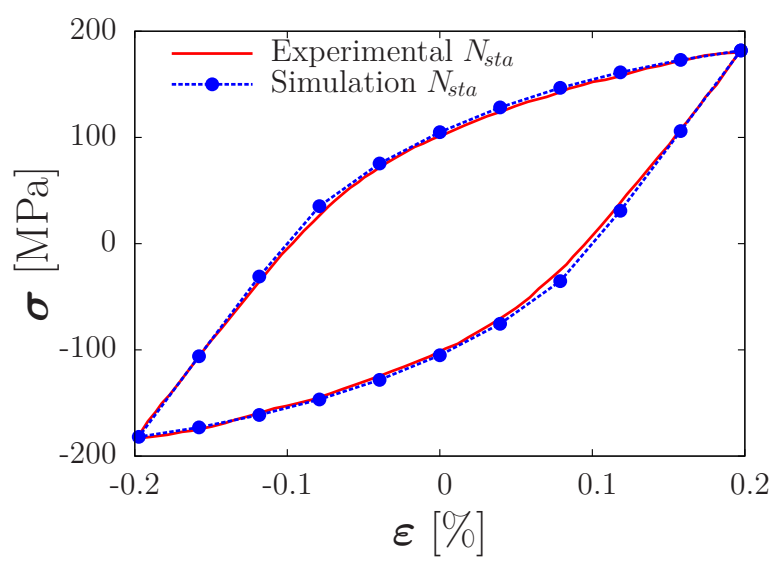

Figure 1: Comparison between experimental and numerical stabilized cycles at $\Delta \varepsilon / 2=0.2 \%$. 


\begin{tabular}{lcccc} 
Mesh & Nodes & Elements & Subdomains & CPU time $[\mathrm{h}]$ \\
\hline Coarse & 23,499 & 16,099 & 1 & 3 \\
Medium & 92,341 & 65,170 & 4 & 20 \\
Fine & 626,899 & 454,673 & 30 & 52
\end{tabular}

Table 2: Nodes, elements, subdomains and CPU time for different mesh densities.

\subsection{Mesh and boundary conditions}

A polycrystalline aggregate mesh of 291 grains is build from a Voronoï tessellation. Mesh dimensions are $250 \mu \mathrm{m} \times 250 \mu \mathrm{m} \times 125 \mu \mathrm{m}$ with an average grain size of $50 \mu \mathrm{m}$. Regularly distributed Voronoï sites map, which would lead to truncated octahedron shaped grains, is used. Then a brownian noise is applied on initial Voronoï sites positions to get quasi homogeneous grain repartition with variable grain boundaries orientations (see figure 2). Free meshing method is applied such that elements conforms grain boundaries. To ensure a good representation of local field heterogeneities, quadratic tetrahedral elements with full integration are used [10]. Three different mesh densities are studied. The finer mesh consists of 626,899 nodes and 454,673 elements, which represent very large numerical cost. In that case, parallel computation is used by means of FETI subdomain decomposition method [23], implemented in Zebulon FE code [24]. The number of nodes, elements, subdomains and CPU time for each mesh density are summarized in table 2

Two cycles are simulated, which is just enough to get information about stress and strain localization. Localization structures are formed in early cycles and get stabilized quickly. Taking account of CPU time in table 2 , cyclic computations on large mesh can be very long, even when restricted to two cycles. Calculating more cycles is incompatible with numerous realizations in order to proceed to a statistical study.

Four types of loading are investigated. We studied two uniaxial cases, loaded in two different directions and two biaxial cases, one with same strain amplitude as uniaxial and one with same equivalent strain amplitude. For each of them, symmetric boundary conditions are applied on aggregate's hidden faces, i.e. opposites of faces $\mathrm{X}, \mathrm{Y}$ and $\mathrm{Z}$ (see figure 2). Face $\mathrm{Z}$, as the component surface, is left free in every cases. The other boundary conditions of each

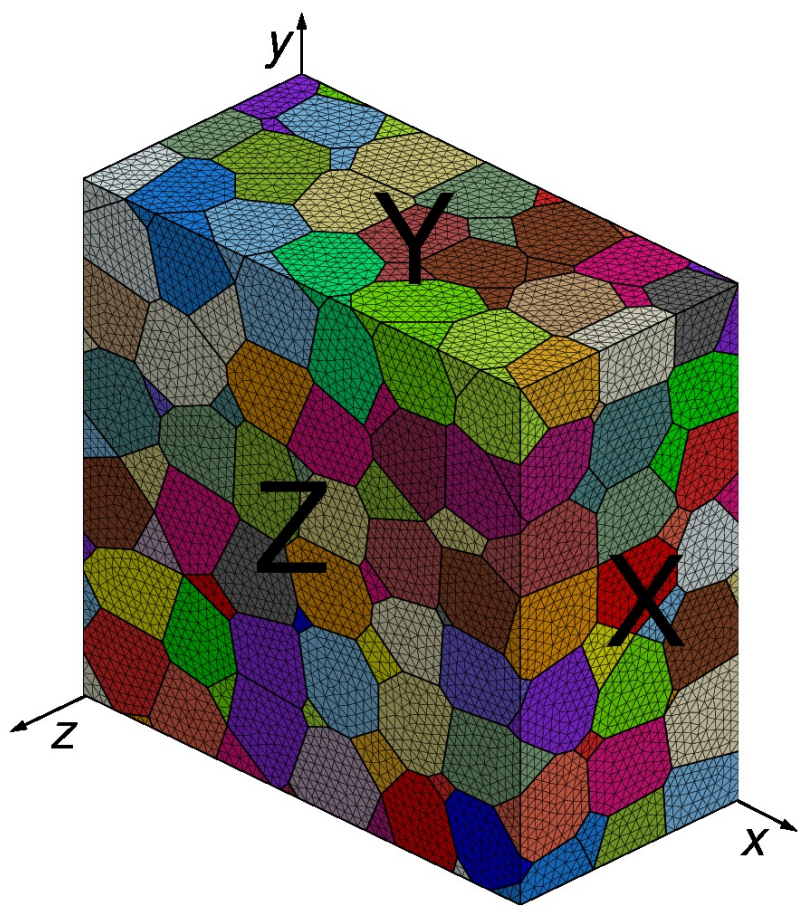

Figure 2: Polycrystalline aggregate mesh of 291 grains with finest density. 
loading are:

(a) Uniaxial-x

- Face X: cyclic displacement to obtain $E_{x x}=$ $\pm 0.2 \%$

- Face Y: uniform normal displacement

(b) Uniaxial-y

- Face X: uniform normal displacement

- Face Y: cyclic displacement to obtain $E_{y y}=$ $\pm 0.2 \%$

(c) Biaxial-eq

- Face X: cyclic displacement to obtain $E_{x x}=$ $\pm 0.14 \%$

- Face Y: cyclic displacement to obtain $E_{y y}=$ $\pm 0.14 \%$

(d) Biaxial

- Face X: cyclic displacement to obtain $E_{x x}=$ $\pm 0.2 \%$

- Face Y: cyclic displacement to obtain $E_{y y}=$ $\pm 0.2 \%$

\subsection{Parameters, variables an scales}

The study is focused on the influence of various local microstructure parameters on the resulting local variables. A relevant grain parameters to characterize mechanical response is the macroscopic Schmid factor $M_{\max }$ calculated with global loading direction $\underline{t}$, slip direction $\underline{l}^{s}$ and slip plane normal $\underset{\sim}{n}$.

$$
M_{\max }=\max _{s}\left|\left(\underline{t} \cdot \underline{l}^{S}\right) \times\left(\underline{t} \cdot \underline{n}^{S}\right)\right|
$$

To take account of neighboring grains, we use a global indicator of misorientation with adjacent grains, noted $\Delta \theta_{w}$. It is provided by summing every grain to grain misorientation weighted by the grain to grain contact surface area.

$$
\Delta \theta_{w}^{i}=\frac{1}{\sum_{j} S^{i, j}} \sum_{j} S^{i, j} \Delta \theta^{i, j}
$$

where $\Delta \theta^{i, j}$ is the misorientation between grain $i$ and $j$, computed according to Randle [25] and taking account of crystalline symmetry.

For the sake of brevity, only plastic strain and stress are reported here, through the sum of cumulated plastic slip $\sum \gamma_{\text {cum }}$ and von Mises equivalent stress $\sigma_{\text {mises }}$.

Different scales results are presented. To get the most local data, we work with Gauss point values. We also study grain averages $\langle\bullet\rangle_{g}$ and grain second order momentums $\langle\bullet\rangle_{g}^{*}$, also called standard deviation, defined as the following:

$$
\begin{gathered}
\langle\bullet\rangle_{g}=\frac{1}{V_{g}} \int_{g} \bullet \mathrm{d} V \\
\langle\bullet\rangle_{g}^{*}=\sqrt{\frac{1}{V_{g}} \int_{g}\left(\bullet-\langle\bullet\rangle_{g}\right)^{2} \mathrm{~d} V}
\end{gathered}
$$

where $V_{g}$ is the total volume of the grain. These two variables give a good representation of mean value and discrepancy inside defined domains.

\section{RESULTS}

All the results presented in this section correspond to the end of the second cycle during the tension phases when the applied strain becomes zero.

\subsection{Effect of mesh density}

Three mesh sizes have been tested to give an idea of the influence of mesh density. Only uniaxial-y loading is considered. Results are studied at two scales: Gauss points values and grain values (average and momentum). Plastic deformation and von Mises stress distributions at the Gauss point scale are shown in figure 3 and related statistical parameters are listed in table 3 At integration point scale, mesh density influences mostly extreme values of local stresses and strains but the effect on aggregate's mean value is negligible. Finest mesh promotes plastic strain localization, mainly near grain boundaries when important strain incompatibilities occur. Thus, a local analysis of polycrystals FE results, i.e at integration points, implies to use homogeneous element size.

Figure 4 reports the grain average values with second order momentum. Here investigations are carried out 


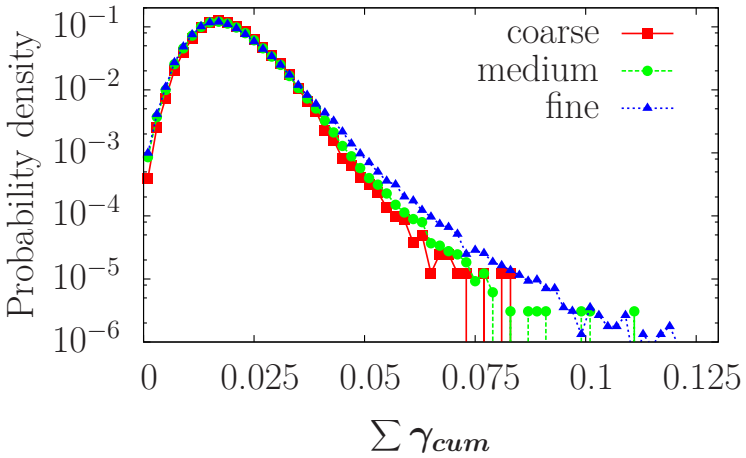

(a)

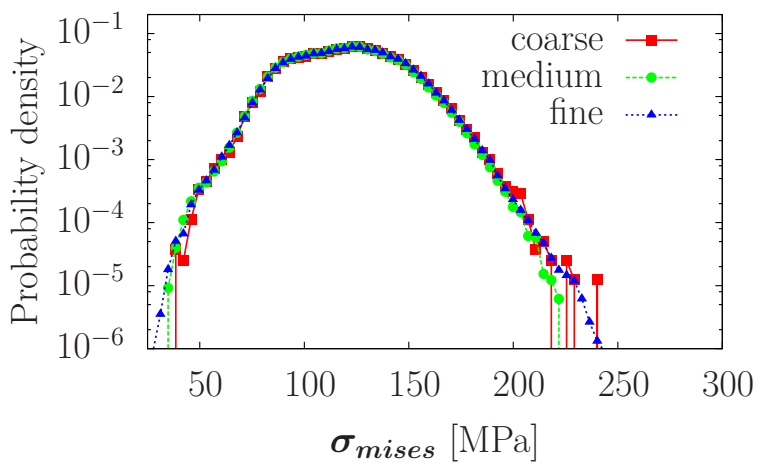

(b)

Figure 3: (a) Plastic strain and (a) von Mises stress distributions at Gauss points depending on mesh density.

\begin{tabular}{lcccc} 
& \multicolumn{4}{c}{$\sum \gamma_{\text {cum }}$} \\
\cline { 2 - 5 } Density & Min & Mean & Max & Std. dev. \\
\hline Coarse & $6.89 \mathrm{e}-07$ & $1.91 \mathrm{e}-02$ & $8.37 \mathrm{e}-02$ & $6.95 \mathrm{e}-03$ \\
Medium & $0.00 \mathrm{e}+00$ & $1.89 \mathrm{e}-02$ & $1.11 \mathrm{e}-01$ & $7.28 \mathrm{e}-03$ \\
Fine & $0.00 \mathrm{e}+00$ & $1.90 \mathrm{e}-02$ & $1.47 \mathrm{e}-01$ & $7.72 \mathrm{e}-03$
\end{tabular}

(a)

\begin{tabular}{lcccc} 
& \multicolumn{4}{c}{$\sigma_{\text {mises }}$} \\
\cline { 2 - 5 } Density & Min & Mean & Max & Std. dev. \\
\hline Coarse & $3.79 \mathrm{e}+01$ & $1.21 \mathrm{e}+02$ & $2.39 \mathrm{e}+02$ & $2.32 \mathrm{e}+01$ \\
Medium & $3.50 \mathrm{e}+01$ & $1.20 \mathrm{e}+02$ & $2.22 \mathrm{e}+02$ & $2.29 \mathrm{e}+01$ \\
Fine & $2.68 \mathrm{e}+01$ & $1.21 \mathrm{e}+02$ & $2.54 \mathrm{e}+02$ & $2.32 \mathrm{e}+01$
\end{tabular}

(b)

Table 3: Statistical data of (a) plastic strain and (a) von Mises stress at Gauss point scale depending on mesh density.

grain per grain, hence mesh density influence is directly characterized on single grain response. Grain labels are sorted by highest plastic strain, respectively stress, obtained with finest mesh computation. Since medium mesh size is used, plastic strain grain averages remain almost the same (see figure 4a). In general the standard deviation inside the grains becomes larger with higher mesh density. Computations with larger meshes are needed to state if local and global values will converge. Nevertheless, to proceed to qualitative studies, our fine mesh is acceptable. Then in the following, every computations are performed with this mesh.

\subsection{Effect of loading}

As explained in section 2.2 four loadings have been applied on the same aggregate. Figure 5 shows the plastic strain isovalues at the free surface of the polycrystal. Plastic strain localization often occurs near grain boundaries and triple points, but also in the core of the grains. Being close to boundary conditions also increases plastic strain. Most of the critical zones, like grain boundaries, are also quite close to the rigid surfaces. Bands oriented at $45^{\circ}$ from loading axis are revealed in both uniaxial- $x$ and uniaxial-y cases (see figure 5a and 5b). Conversely, when biaxial or biaxial-eq loadings are applied, these bands vanished (see figure 5c and 5d). In biaxial case, intense 


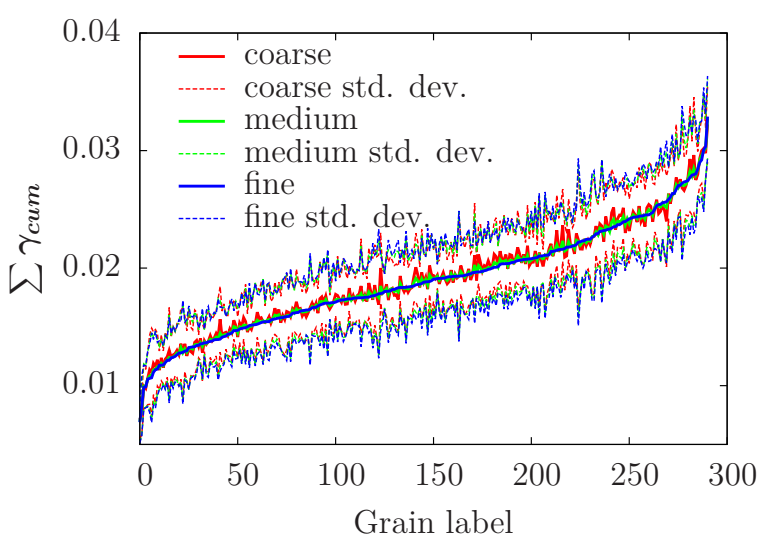

(a)

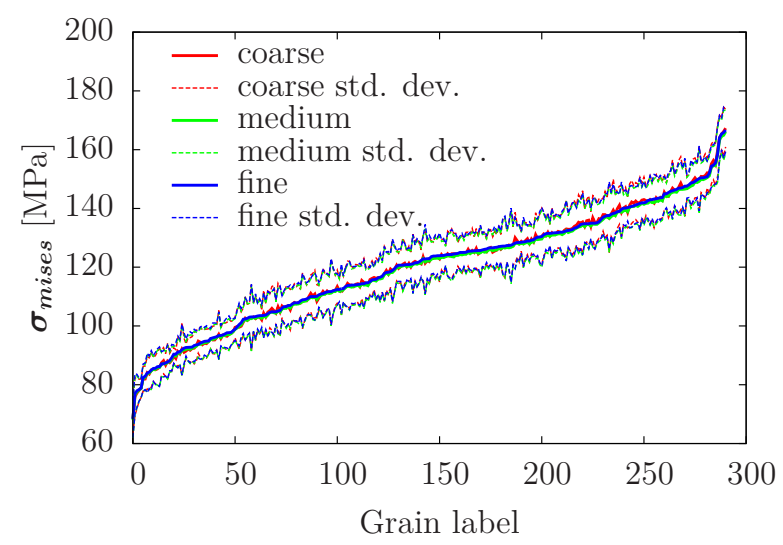

(b)

Figure 4: Grain averages and dispersion of (a) plastic strain and (b) von Mises stress depending on mesh density.

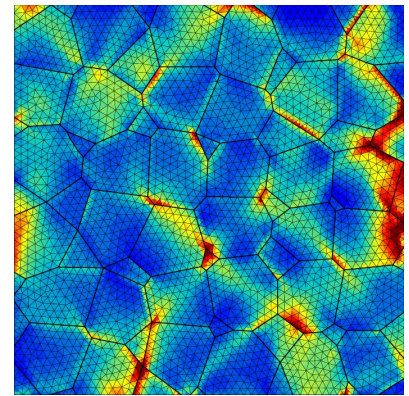

(a) Uniaxial-x

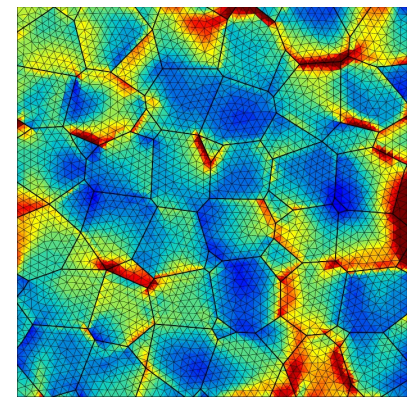

(c) Biaxial-eq

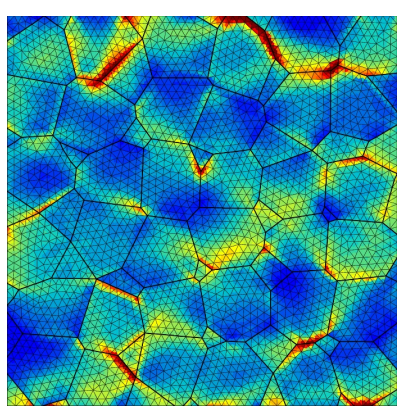

(b) Uniaxial-y

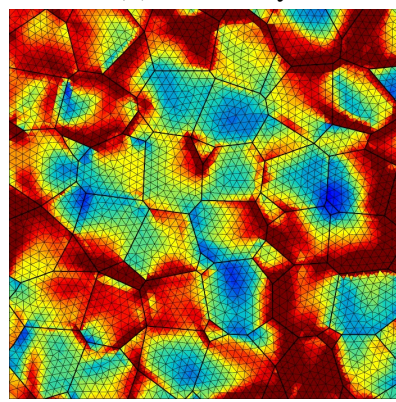

(d) Biaxial

\section{$\begin{array}{lllll}0 & 0.015 & 0.03 & 0.045 & 0.06\end{array}$}

Figure 5: $\sum \gamma_{\text {cum }}$ map at free surface $\mathrm{Z}$ for each loading case.

plastic zones are not always the combination of the ones identified in both uniaxial cases. With the same equivalent applied strain amplitude, plastic strain is a bit higher with biaxial loading (see figures $5 \mathrm{a}$ and $5 \mathrm{c}$ ). Hence, that kind of loading conditions is more critical. However, surface investigations are not sufficient to completely characterize strain localization intensity and morphology.

The influence of loading conditions can also be seen at the global scale where localization bands expand through several grains. Using transparency filter, it is possible to give a global view of plastic deformation localization inside the aggregate. Plastic deformation isovalues, seen in every space direction, are displayed in figure 7 under uniaxial-y and biaxial loadings. Localization bands are oriented $45^{\circ}$ with respect to the loading direction in each cases but they do not correspond to the same type of facets as Miller introduced earlier [26]. These two types of facets are explained in figure 6. Taking account of slip 

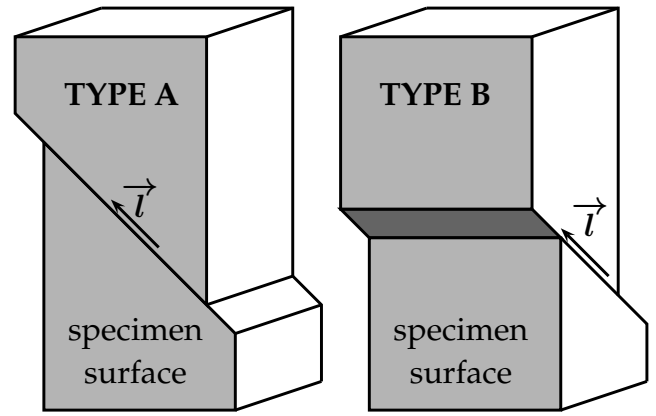

Figure 6: Facets of type A and B, according to Miller [26].

direction $\underline{l}$, only B-type facets are emerging to the surface, leading to surface extrusions. Their path can be related to stage 1 fatigue cracks growing in the specimen core. Atype facets have no component in the free surface direction, thus they propagate along the surface and can hardly emerge at the surface.

In the uniaxial case (see figure $7 \mathrm{a}$ ), $45^{\circ}$ bands appear in $X$ (type $\mathrm{A}$ ) and $Z$ (type $\mathrm{B}$ ) views. Then in the biaxial case (see figure 7b), B-type facets expand when looking in $X$ and $Y$ direction. Under these loading conditions, no A-type facets are created. We can deduce that biaxial loading can lead to an earlier fatigue crack initiation at the surface.

\subsection{Statistical analysis}

15 computations have been performed on the same mesh but with different orientation sets. Many Schmid factors associated with various misorientation configurations are therefore tested. Figure 8 shows the average $\sum \gamma_{\text {cum }}$ and $\sigma_{\text {mises }}$ over every simulation depending on local microstructure parameters $M_{\max }$ and $\Delta \theta_{w}$. The plots are realized for different types of grains, whether they are inside the core of the aggregate, at the free surface or involved in Boundary Conditions (BC).

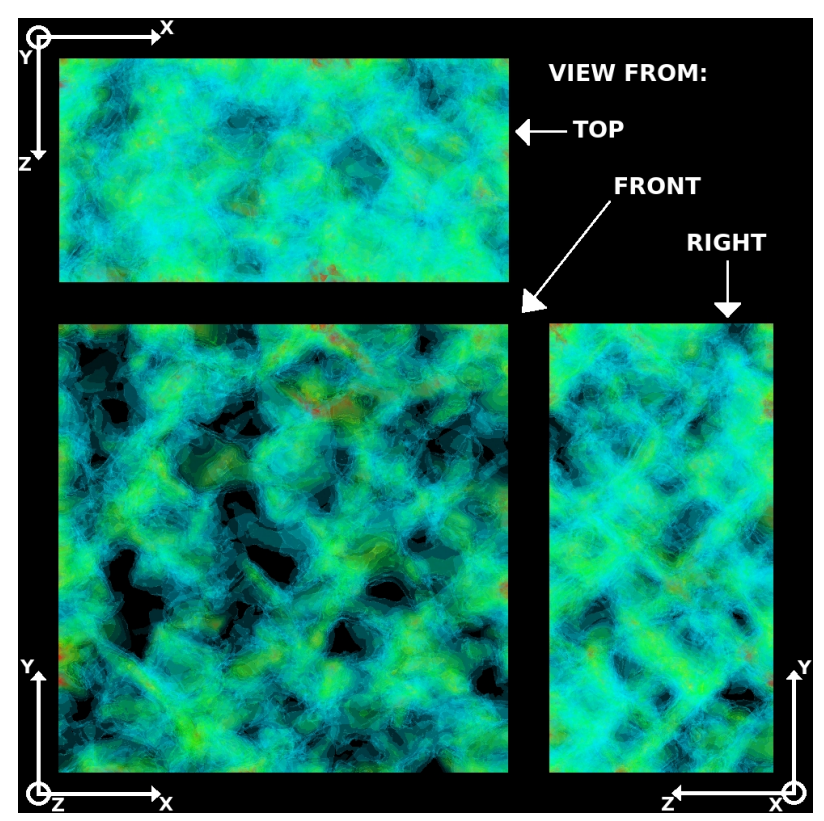

(a)

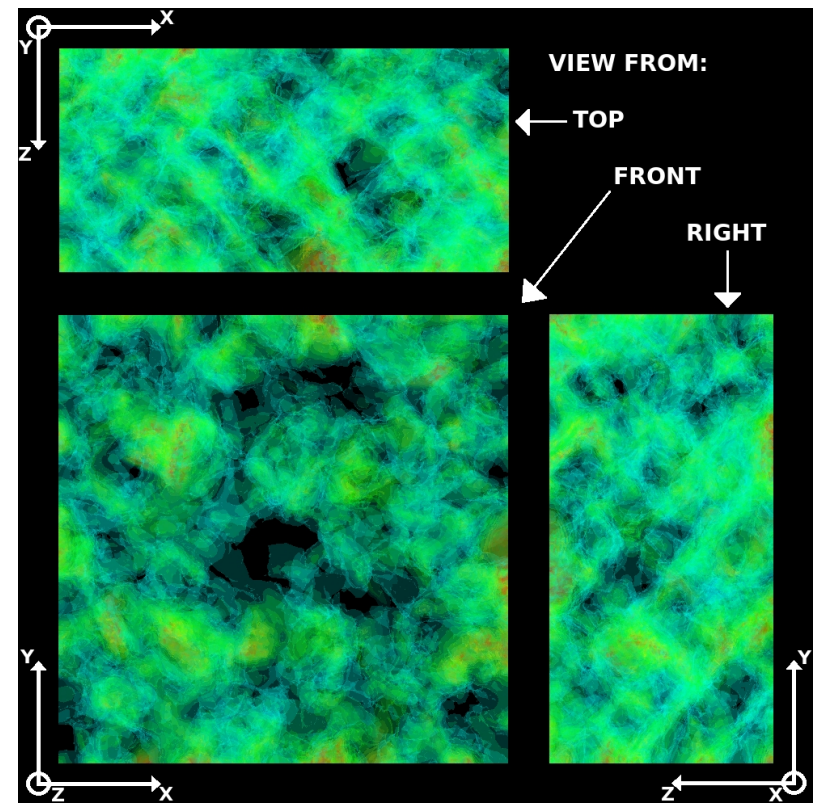

(b)

Figure 7: Transparent volumetric views of $\sum \gamma_{\text {cum }}$ for (a) uniaxial-y and (b) biaxial-eq loadings. 


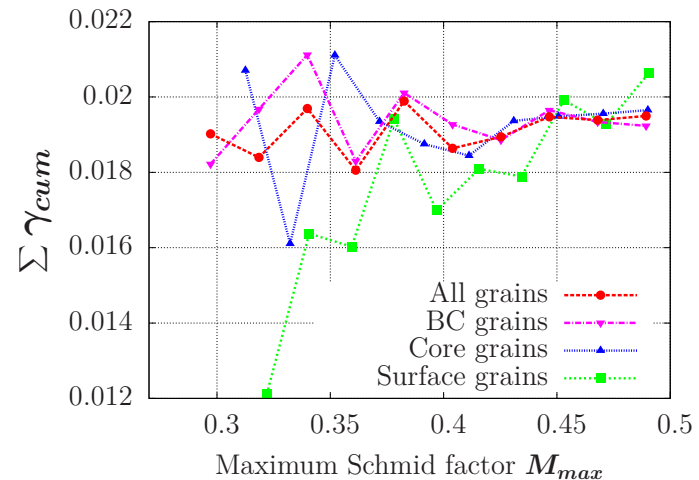

(a)

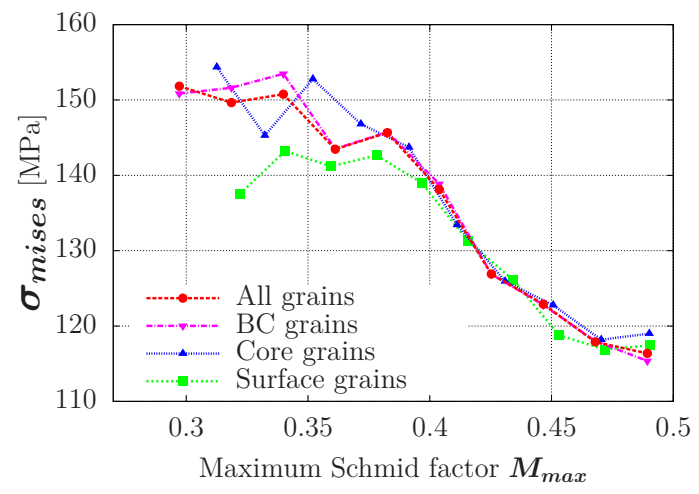

(b)

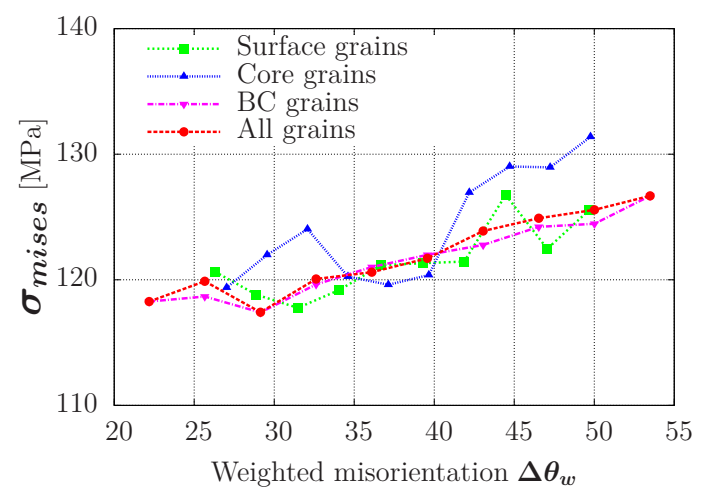

(c)

Figure 8: Influence of Schmid Factor on (a) $\sum \gamma_{\text {cum }}$ and (b) $\sigma_{\text {mises }}$ for different types of grains. (c) Influence of weighted misorientation $\Delta \theta_{w}$ on $\sigma_{\text {mises }}$.
Most of the grains, 192 among the 291, which is more than $65 \%$, are directly affected by $\mathrm{BC}$ and therefore cannot be considered as effective polycrystal behavior. As a consequence, the overall grain behavior is closer to $\mathrm{BC}$ grains. 58 grains are in the core and 51 are at the surface far from BC (separated by at least 1 grain). Only a third of computed grains of the simulation are workable, so more computations may be needed to give clearer results. Using periodic boundary conditions to reproduce semi-infinite medium, would also be a solution to avoid this problem.

The Schmid factor is easily correlated to the amount of plastic shear in surface grains, which behavior is closer to single crystal due to free surface (see figure 8a). Core grains are more influenced by surrounding grains and consequently are more subject to neighboring effect. On the contrary, high Schmid factor grains are less constrained (see figure 8b). Earlier numerical study already exhibits the same tendency [12]. Higher misorientation slightly increases the stress inside the grains, but by a very weak ratio (figure 8c). This effect seems stronger for core grains, almost $+1 \mathrm{MPa} /{ }^{\circ}$ against $+0.3 \mathrm{MPa} /{ }^{\circ}$ for surface grains.

\subsection{Influence of surface roughness}

A rough surface is introduced explicitly in the mesh. A part of an experimental 1D roughness profile has been extended to produce a surface. Then it is applied by node displacement method until a fixed depth with a linear decrease. Figure 9 shows the original one-dimensional roughness profile, the semi-synthetic surface roughness, the resulting mesh, plastic strain maps (in both flat and rough cases) and surface topography (to locate the valleys). The solid space in figure 9a corresponds to the selected part of the profile.

Plastic strain localization that occurs in control case is only due to crystallographic orientation. In the rough aggregate case, localization emanating from grain orientation is only partial. Geometric singularities have a real impact on free surface localization patterns. Critical plastic zones follow relief valleys. Because real life components are not perfectly polished, flat aggregate computations are not fully relevant, at least for studies focused on surface grains. Deepest analysis, not presented here, shows that this roughness does not change the results under the first layer of grains. Although surface roughness can mod- 
ify crack initiation prediction, the possibility of crossing the first grain boundaries should remain unchanged. To go further, surface strengthening, residual stresses and recrystallization generated by industrial forming process should also be introduced in that kind of simulation.

\section{CONCLUSIONS}

Three dimensional FE computations of synthetic polycrystalline aggregate composed of 291 grains have been performed using crystal plasticity model. A first outline of microstructure features implication on local plasticity has been given with a statistical analysis. Investigations were carried out with various mesh densities, loadings, orientation sets and surface roughnesses. Many conclusions can be made:

i Mesh density can affect results, mainly the extreme values with very coarse meshes. Grain averages are more stable, but finest mesh increases dispersion inside individual grains.

ii Biaxial loading, even with same equivalent strain amplitude, produces more plastic strain. Furthermore, the localization structures remind Miller's Btype facets, which produce surface extrusions and propagate in the inside of the component.

iii The Schmid factor characterizes well the vulnerability of surface grains. Core grains are more prone to neighboring effect.

iv Finally, adding a rough surface changes drastically plasticity patterns at the free surface, but only on the first layer of grains. Hence it decreases the role of crystallographic orientation on crack initiation at the grain scale. The micro-propagation through microstructural barriers should not be affected by geometric singularities at the free surface.

\section{ACKNOWLEDGMENT}

The authors would like to express their thanks to ANR project AFGRAP for financial support.

\section{References}

[1] U. Essmann, U. Gösele, and H. Mughrabi. A model of extrusions and intrusions in fatigued metals: I. Point-defect production and the growth of extrusions. Phil. Mag., 44:405-426, 1981.

[2] B.-T. Ma and C. Laird. Overview of fatigue behavior in copper single crystals: I. Surface morphology and stage I crack initiation sites for tests at constant strain amplitude. Acta Mat., 37:325-336, 1989.

[3] C. Blochwitz, J. Brechbühl, and W. Tirschler. Analysis of activated slip systems in fatigue nickel polycrystals using the EBSD-technique in the scanning electron microscope. Mater. Sci. Eng., A210:42-47, 1996.

[4] C. Blochwitz, R. Richter, W. Tirschler, and K. Obrtlík. The effect of local textures on microcrack propagation in fatigued F.C.C. metals. Mater. Sci. Eng., A234-236:563-566, 1997.

[5] J. Man, K. Obrtlík, C. Blochwitz, and J. Polák. Atomic force microscopy of surface relief in individual grains of fatigued $316 \mathrm{~L}$ austenitic stainless steel. Acta Mat., 50:3767-3780, 2002.

[6] J. Man, K. Ortblík, and J. Polák. Study of surface relief evolution in fatigued $316 \mathrm{~L}$ austenitic stainless steel by AFM. Mater. Sci. Eng., A351:123-132, 2003.

[7] A. King, G. Johnson, D. Engelberg, W. Ludwig, and J. Marrow. Observations of intergranular stress corrosion cracking in a grain-mapped polycrystal. Science, 321(5887):382-385, 2008.

[8] M. Herbig, A. King, P. Reischig, H. Proudhon, E.M. Lauridsen, J. Marrow, J.-Y. Buffière, and W. Ludwig. 3-D growth of a short fatigue crack within a polycrystalline microstructure studied using combined diffraction and phase-contrast X-ray tomography. Acta Mat., 59:590-601, 2011.

[9] C. Déprés, C.F. Robertson, and M.C. Fivel. Crack initiation in fatigue: experiments and threedimensional dislocations simulations. Mater. Sci. Eng., A387-389:288-291, 2004. 


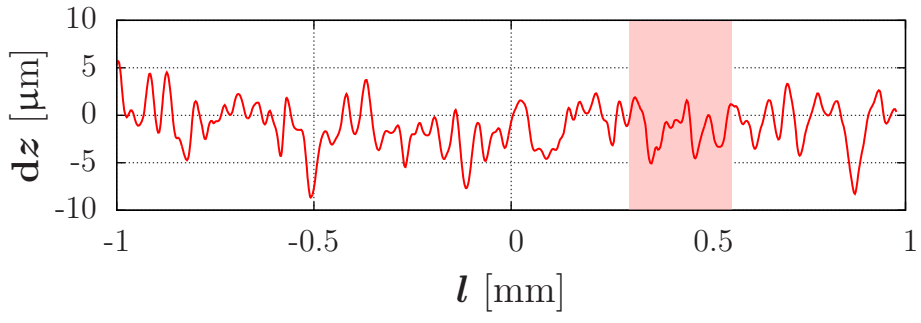

(a)

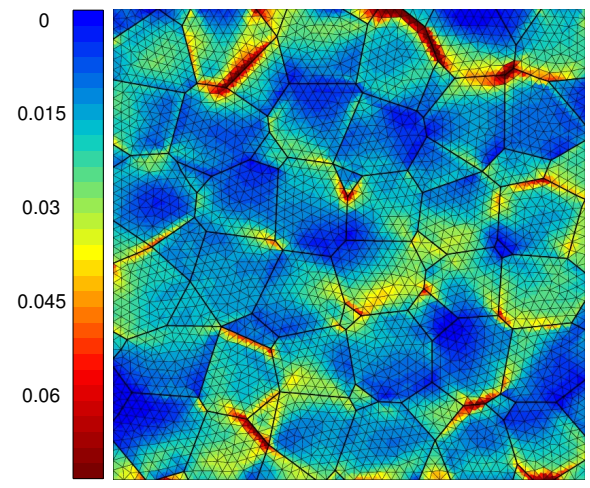

(c)

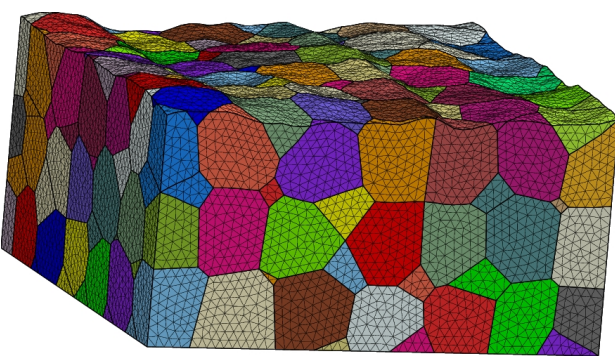

(b)

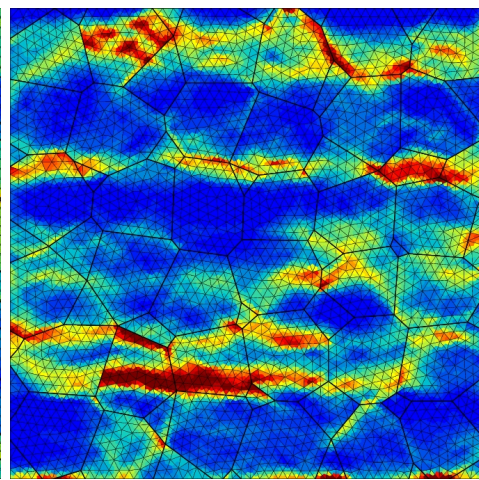

(d)

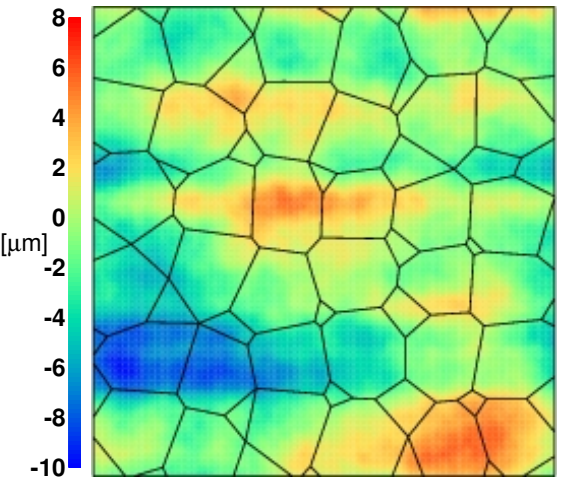

(e)

Figure 9: (a) 1-D roughness profile, (b) application to mesh, resulting $\sum \gamma_{\text {cum }}$ map for (c) control case with flat mesh and for (d) rough case. (e) Surface topography. 
[10] F. Barbe, L. Decker, D. Jeulin, and G. Cailletaud. Intergranular and intragranular behavior of polycrystalline aggregates. Part I: FE model. Int. J. of Plasticity, 17(4):513-536, 2001.

[11] F. Barbe, S. Forest, and G. Cailletaud. Intergranular and intragranular behavior of polycrystalline aggregates. Part II: Results. Int. J. of Plasticity, 17(4):537-563, 2001.

[12] Y. Guilhem, S. Basseville, F. Curtit, J-M. Stéphan, and G. Cailletaud. Investigation of the effect of grain clusters on fatigue crack initiation in polycrystals. Int. J. Fatigue, 32:1748-1763, 2010.

[13] I. Simonovski and L. Cizelj. The influence of grain's crystallographic orientations on advancing short crack. Int. J. Fatigue, 29:2005-2014, 2007.

[14] I. Simonovski and L. Cizelj. Grain scale model of small cracks. In 15th International Conference on Nuclear Engineering, Nagoya, Japan, April 2007.

[15] C.P. Przybyla and D.L. McDowell. Microstructuresensitive extreme value probabilities for high cycle fatigue of Ni-base superalloy IN100. Int. J. of Plasticity, 26:372-394, 2010.

[16] L. Méric and G. Cailletaud. Single crystal modeling for structural calculations. Part 2: Finite element implementation. J. of Engng. Mat. Technol., 113:171$182,1991$.

[17] M. Sauzay. Effet de l'anisotropie élastique cristalline sur la distribution des facteurs de Schmid à la surface des polycristaux. C.R. Mécanique, 334:353-361, 2006.

[18] P. Franciosi. The concepts of latent hardening and strain hardening in metallic single crystals. Acta Metall., 33:1601-1612, 1985.

[19] C. Gérard, B. Bacroix, M. Bornert, G. Cailletaud, J. Crépin, and S. Leclercq. Hardening description for FCC materials under complex loading paths. Computational Materials Science, 45:751755, 2009.
[20] S. Queyreau, G. Monnet, and B. Devincre. Slip systems interactions in $\alpha$-iron determined by dislocation dynamics simulations. Int. J. of Plasticity, 25:361-377, 2009.

[21] G. Cailletaud and P. Pilvin. Utilisation de modèles polycristallins pour le calcul par éléments finis. Revue Européenne des Éléments Finis, 3(4):515-541, 1994.

[22] G. Cailletaud. A micromechanical approach to inelastic behaviour of metals. Int. J. of Plasticity, 8:55-73, 1992.

[23] C. Farhat and F.-X. Roux. Implicit parallel processing in structural mechanics. Computational Mechanics Advances, 2(1):1-124, 1994.

[24] F. Feyel. Application du calcul parallèle aux modèles à grand nombre de variables internes. $\mathrm{PhD}$ thesis, École Nationale Supérieure des Mines de Paris, 1998.

[25] V. Randle. Microtexture Determination and its Applications. The Institute of Materials, 1992.

[26] K.J. Miller. The behaviour of short fatigue cracks and their initiation. Part II-A General summary. Fatigue and Fracture of Engng Mat and Struct, 10:93113, 1987. 